both day 14 and day 28 of post induction. Spleen mass were significantly increased in LILRA3 knock-in mice $(p<0.001)$. The proportion of Th2, TFH, germinal center (GC) B, and plasma $B$ cells were increased in knock-in mice $(p<0.01)$, but not Th17 and regulatory $\mathrm{T}$ cells. Concentration of serum antidsDNA IgG was significantly elevated in knock-in mice $(\mathrm{p}<0.001)$.

Conclusions Our data indicate that LILRA3 promotes lupuslike disease probably through the excessive expression of Tfh cells and GC B cells, subsequently help for the induction and maintenance of plasma cell differentiation and autoantibody production.

Funding Source(s): None

\section{PATTERNS OF ORGAN INVOLVEMENT IN SLE AND THEIR OUTCOME: A REAL LIFE EXPERIENCE IN A LUPUS CLINIC}

${ }^{1}$ Md Mujibur Rahman*, 'Md Moniruzzan, 'Jakaria Been Sayeed, ${ }^{2}$ Rashedul Hassan, ${ }^{3}$ Satyajit Barua, ${ }^{4}$ Shaoun Barua, ${ }^{1}$ Md Uzzwal Mallik, ${ }^{4}$ Abdur Rahim. ${ }^{1}$ Dhaka Medical College; ${ }^{2}$ Green Life Medical College, Dhaka; ${ }^{3}$ Shaheed Suhrawardy Medical College Hospital; ${ }^{4}$ Shaheed Suhrawardi Medical College

\subsection{6/lupus-2019-Ism.239}

Background Systemic lupus erythematosus (SLE) is a multisystem autoimmune disorder predominantly affecting the women of childbearing age. It often manifests with various constitutional symptoms as well as combination of organ systems involvement and outcome varies in different population with available treatment. The present study is to see the patterns of organ involvement and their outcomes at first 6 months with standard treatment.

Methods This was a retrospective study done in lupus clinic of Shaheed Suhrawardy Medical College Hospital, Dhaka, Bangladesh during 2010 to 2016. Patients were included based on ACR lupus diagnostic criteria and had received standard treatment. Outcomes were assessed regularly by clinical features, urinalysis and serum creatinine done in the appropriate cases. Composite endpoint was calculated by using SELENA SLEDAI in all the cases.

Results Among 120 patients, 111 (92.5\%) were female, age ranging from 14 to 57 years with a mean of $26.5 \pm 10.68$ (SD) year. Median follow up was 1.7 years. The most common manifestations were fever $(70 \%)$, joint pain $(72.5 \%)$, oral ulceration (49\%), alopecia (40\%), malar rash (28.3\%), photosensitivity $(25.8 \%)$ and Raynauds phenomenon (19\%). Commonly involved major organ-systems were renal (40\%), neuropsychiatric (19\%), respiratory (14\%), cardiac (6.6\%) and hematological (6.6\%). In lupus nephritis, proteinuria was present in $100 \%$ of cases. The other parameters of renal involvements were $\mathrm{RBC}>5 / \mathrm{HPF}$ and $\mathrm{RBC}$ or cellular cast in $37.5 \%$. Serum creatinine was raised in 23 patients $(19.17 \%)$ with a mean of $1.68 \pm 0.96 \mathrm{mg} / \mathrm{dl}$. Renal biopsy was done in $40(83.3 \%)$ cases. The histology showed class-II in $03(7.5 \%)$, Class-III in $03(7.5 \%)$, class-IV in $20(50 \%)$ and class-V in 08 $(20 \%)$ cases. With standard treatment, major reduction of mean serum creatinine, 24 hours UTP and SLEDAI were observed in most of the cases at least after 6 months. Total flare occurred in $31(25.8 \%)$ among them renal flare was in $10(8.33 \%)$ and non-renal flare in 21 (17.5\%). Four SLE patients died during the course of treatment.
Conclusions Renal and central nervous systems are the most commonly involved major organ systems. The overall outcome is favorable with standard treatment.

Funding Source(s): None

\section{TRANS-ANCESTRAL EXCLUSION MAPPING STRATEGY PRIORITISES RISK ALLELES AT IKZF3 WITH INCREASED BIOLOGICAL RELEVANCE}

${ }^{1}$ Deborah S Cunninghame Graham*, SLE Immunochip Consortium, ${ }^{2}$ Timothy Vyse. ${ }^{1} \mathrm{KCL}$; ${ }^{2}$ King's College London

10.1136/lupus-2019-Ism.240

Background Pinpointing causal risk alleles, carried on extended risk haplotypes, at susceptibility loci for common disease is challenging. We present a novel strategy to prioritise tag-SNPs at risk loci with the highest likelihood of biological relevance for laboratory functional studies. This strategy involves transancestral mapping of risk-haplotypes, MAF-exclusion-mapping of discordant variants, followed by co-localisation of tag-SNPs with epigenetic annotation/identification of allele-specific transcription factor (TF) binding sites, using data from public databases such as RoadMap, ENCODE and HaploRegv4, as a surrogate for causality.

We illustrate the utility of this approach at the Ikaros zinc finger TF IKZF3, with a $194 \mathrm{~kb}$ associated European (EUR) SLE haplotype carrying 282 tag-SNPs, extending over multiple genes from the IKZF3 3 flanking-region into the upstreamregion of ORMDL3.

Methods Align IKZF3 control haplotypes from our large EUR GWAS and five 1000G super-populations. Compare EUR-African MAF to delineate discordant tag-SNPs. Use SLE ImmunoChip data to exclude variants exhibiting $>3 \% \mathrm{MAF}$ and not associated $(\mathrm{p}>0.05)$ in African-Americans (AA). Undertake colocalisation analysis, using multiple tools (Coloc-stats webserver), to prioritise risk alleles overlapping DNAse I hotspots and chromatin modifications characterising active enhancers and/or promoters in blood cell-types (RoadMap data). Additional evidence of potential function was sought for chromatin looping (3D Genome Browser) and differential TF binding (HaploRegv4).

Results Trans-ancestral mapping reduced the risk-haplotype by $47 \%$ to $101 \mathrm{~kb}$ and the tag-SNPs by $28 \%$ to 140.26 tagSNPs exhibited association in both EUR and AA SLE ImmunoChip cohorts. All 26 variants reside in regions of open chromatin in LCLs. 15 of the tag-SNPs lie within regions involved in chromatin-looping events, bringing together the full-length promoter and I1 of a shorter IKZF3 isoform. We discovered allele-specific binding of Fox family members to risk alleles in the promoter (rs111678394) and variants in I1 of the shorter isoform: rs113730542 and rs112876941.

Conclusions We hypothesise that allele-specific binding of Fox TFs to risk alleles in the promoter and/or I1 of the shorter isoform IKZF3 may independently modulate the expression of both isoforms. Since the shorter isoform lacks the four zinc fingers responsible for DNA binding of Aiolos (E4-6), the shorter isoform is unable to directly bind DNA. Heterodimerisation between the two isoforms may therefore sequester the full-length isoform in a biologically inactive form. If Fox TFs stabilise chromatin loops within IKZF3 in an allele-specific manner, this may provide a mechanism where risk alleles 
influence the activity/availability of IKZF3 and promote autoimmunity.

Funding Source(s): Versus Arthritis, formerly Arthritis Research UK project grant (20265)

\section{SINGLE CELL GENOMICS OF SELF-REACTIVE B CELLS REVEALS THE EVOLUTION FROM BENIGN TO PATHOGENIC AUTOANTIBODY AND STRATEGIES FOR EARLY DIAGNOSIS AND PERSONALISED TREATMENT}

\footnotetext{
${ }^{1}$ Mandeep Singh, ${ }^{1}$ Katherine Jackson, ${ }^{2}$ Jing Jing Wang, ${ }^{1}$ Peter Schofield, ${ }^{3}$ Matt Field, ${ }^{1}$ Timothy Peters, ${ }^{4}$ Fabio Luciani, ${ }^{2}$ Tom Gordon, ${ }^{1}$ Christopher Goodnow, ${ }^{1}$ Joanne H Reed* ${ }^{1}$ Garvan Institute of Medical Research; ${ }^{2}$ Flinders University of South Australia; ${ }^{3}$ Australian Institute of Tropical Health and Medicine, James Cook University; ${ }^{4}$ Kirby Institute, UNSW
}

\subsection{6/lupus-2019-Ism.241}

Background Autoantibodies appear in the serum years before the development of clinical disease in patients ultimately diagnosed with systemic lupus erythematosus (SLE) or Sjögrens syndrome. The transition from benign to pathogenic autoimmunity is not understood. Anti-IgG rheumatoid factors provide an illuminating example of an autoantibody that persists, on average, more than four years prior to the development of systemic vasculitis and type II cryoglobuinemia. The aim of this study was to identify the pathogenic changes occurring in B cells making rheumatoid factor autoantibody during this transition.

Methods Massively parallel sequencing of peripheral blood B cell receptors (BCR) was combined with mass spectrometry peptide sequencing of serum rheumatoid factor autoantibodies to identify circulating B cells expressing IgM rheumatoid factors in 4 patients with SLE or Sjögrens syndrome. In a patient presenting with primary Sjögrens syndrome evolving to cutaneous vasculitis, blood samples before and after transition were subjected to single cell analysis of mRNA, genome methylation, and lymphocyte regulatory genes recurrently mutated in lymphoma and leukemia.

Results Circulating rheumatoid factor B cell clones and their matched serum autoantibodies were identified in 4 patients, 3 of whom, produced the stereotypic Wa-type rheumatoid factor with IGHV1-69 and IGKV3-20 variable domains. Longitudinal analysis of a patient before and after vasculitis revealed a single Wa-type rogue clone was responsible for the rheumatoid factor over a period of 6 years. Compared to the patients normal memory $\mathrm{B}$ cells, the rogue cells had aberrant gene expression corresponding to CD11c+CD21 low B cells observed in autoimmunity, and hypomethylation of gene regions aberrantly hypomethylated in chronic lymphocytic leukemia (CLL). A loss-of-function somatic mutation in a Kelch-like protein gene, KLHL6 identical to recurring CLL and lymphoma mutations was acquired by half of the rheumatoid factor B cells. KLHL6 mutant cells slowly accumulated antibody variable domain replacement mutations that did not alter binding affinity but caused IgM-IgG immune complexes to precipitate at temperatures below 25C

Conclusions Transition from a benign to pathogenic rheumatoid factor was preceded by a lymphoproliferative disease mutation in the responsible $\mathrm{B}$ cells and resulted in accumulation of antibody somatic mutations that diminish immune complex solubility. The ability to detect clones of the rheumatoid factor that are accumulating replacement mutations years prior to the patient developing cryoglobulinemia represents an opportunity to interfere with the conversion from asymptomatic seropositivity to clinical disease.

Funding Source(s): Australian National Health and Medical Research Council and New South Wales Department of Health

\section{ROLE OF STIGMA AND SOCIAL ISOLATION ON DEPRESSION IN PATIENTS WITH CHRONIC CUTANEOUS LUPUS ERYTHEMATOSUS}

Cristina Drenkard*, Laura Aspey, Gaobin Bao, Charmayne Dunlop-Thomas, S Sam Lim. Emory University

\subsection{6/lupus-2019-Ism.242}

Background Skin disorders can cause mental health problems associated with stigmatization and social isolation. We found that $26 \%$ of individuals with chronic cutaneous lupus erythematosus (CCLE) reported moderate to severe depressive symptoms. While patients perceptions of disrespectful office staff increased the risk of depression, emotional support was associated with a reduction. In this study, we examined the relationships between stigma, social isolation, and depression. We further examined if social isolation mediates the relationship between stigma and depression and whether sociodemographic characteristics may moderate those relationships.

Methods We conducted a cross-sectional study in a predominantly African American cohort of patients with primary CCLE from metropolitan Atlanta, Georgia, U.S. Depression, stigma and social isolation were assessed using the NIH PROMIS short forms. Linear and multiple regression were used to examine the relationship between depression, stigma and social isolation. Education attainment, poverty and race were explored as potential moderators.

Results Among 118 patients with a documented diagnosis of primary CCLE, 104 (88\%) were female, 96 (81\%) were African American, 54 (46.2\%) completed high school or less, $40(40.8 \%)$ lived in poverty and 34 $(28.8 \%)$ self-reported moderate to severe depressive

Abstract 242 Table 1 Multiple linear regression models for predicting depression in CCLE

\begin{tabular}{|c|c|c|c|c|}
\hline \multirow[b]{2}{*}{ Predictor Variable } & \multicolumn{2}{|c|}{$\begin{array}{l}\text { Model } 1^{*} \\
\text { (Mediator effect of Social } \\
\text { Isolation) }\end{array}$} & \multicolumn{2}{|c|}{$\begin{array}{l}\text { Model } 2^{* *} \\
\text { (Moderator effect of } \\
\text { Education) }\end{array}$} \\
\hline & $\beta$ & $p$-value & $\beta$ & $\mathrm{p}$-value \\
\hline Stigma & -0.018 & 0.88 & 0.270 & 0.011 \\
\hline Social isolation & 0.527 & $<0.0001$ & & \\
\hline Education ( $\leq$ high school) & - & - & -22.30 & 0.009 \\
\hline Stigma*Education ( $\leq$ high school) & - & - & 0.454 & 0.004 \\
\hline
\end{tabular}

*Controlled for age, race, gender and education; ** Controlled for age, race and gender 